\title{
Antropometría del Estribo en Humanos: Un Estudio Anatómico Directo
}

\author{
Human's Stape Anthropometry: A Direct Anatomic Study
}

Ramirez, L. M. \& Ballesteros, L. E.

RAMIREZ, L. M. \& BALLESTEROS, L. E. Antropometría del estribo en humanos. Un estudio anatómico directo. Int. J. Morphol., 30(4):1295-1300, 2012.

RESUMEN: Es modesta la base de datos morfológicos del estribo en humanos. Su caracterización morfométrica es importante cuando se compara con estribos de otras épocas ancestrales e igualmente en el estudio de una biomecánica oscicular compleja. Las características estructurales y dimensionales y pareadas del estribo fueron registradas en 23 huesos de población mestiza adulta Colombiana. Las medidas registradas involucraron el área de la articulación lenticular incudo-estapedial, la platina del estribo y los procesos anterior y posterior del estribo, en diferentes sentidos. Cinco de los dieciséis registros tomados en este estudio pudieron ser comparados concienzudamente con otros estudios. Las cinco medidas comparables fueron: el largo total de la platina con 2,87 mm DE 0,09; el ancho mayor de la platina con 1,36 mm DE 0,09 ; el largo del estribo con 3,18 DE 0,15 y la distancia interna interpilar con 1,65 DE 0,17 ; y la altura del arco con 1,82 DE 0,19. La angulación de los procesos anterior y posterior del estribo resulto en 29.01 grados DE 2.93. Conforme a la población estudiada no se logro encontrar variación individual o pareada en la anatomía del estribo, haciéndolo un hueso morfométricamente uniforme y estable.

PALABRAS CLAVE: Oscículos, estribos, platina, articulación lenticular, articulación incudo-estapedial, proceso anterior del estribo, proceso posterior del estribo.

\section{INTRODUCCIÓN}

Fue Andrew Vesalius (Cole, 1949) en el siglo XVI quien inicio el registro en oscículos, sin embargo es insuficiente el material a la fecha. Adicional al valor morfométrico de su registro, es valiosa la información en procedimientos quirúrgicos otológicos y útil en modelos que estudian su funcionamiento (Willi et al., 2002).

Los huesos del oído medio comienzan su osificación en la zona dorsal del primer arco branquial y complementariamente del segundo arco branquial terminando sus dimensiones finales intrauterinamente (Rodriguez Vazquez et al., 1991; Rodriguez Vazquez et al., 1993; Nishizaki \& Anniko, 1997; Whyte et al., 2002; Whyte et al., 2003). Se unen en articulaciones diartrosicas y logran superar la impedancia acústica que genera la energía sonora cuando pasa de aire a líquido (Olsewski, 1989; Olsewski, 1990; Guyton \& Hall, 2007). Estas estructuras óseas son irregulares e imbalanceadas, lo que genera efecto de palanca mecánica en la transmisión sonora debido a los momentos de fuerza que esta morfología genera (Ramirez \& Ballesteros, 2010). El final de toda esta mecánica impulsa la platina sobre la ventana oval de la coclea, transmitiendo la energía sobre perilinfa y endolinfa que últimamente desplaza las células ciliadas para el primer paso de una mecano-transducción hacia diferentes niveles centrales que terminan en la corteza auditiva (Gillespie \& Muller, 2009).

Los huesecillos del oído, incluido el estribo, han sido estudiados en poblaciones prehistóricas e históricas de diferentes orígenes étnicos y geográficos que han corroborado su estabilidad morfométrica y mínima variabilidad (Heron, 1923; Harneja \& Chaturvedi, 1973; Arensburg et al., 1980; Moggi-Cecchi \& Collard, 1992; Unur et al., 2002). La población en este trabajo esta conformada por muestras de mestizos Colombianos en los que se busca encontrar variaciones.

* MS, MSc. Profesor Asociado Universidad de Antioquia. Medellín, Colombia. Grupo Estomatología Biomédica.

**MD, MSc. Profesor Titular Universidad Industrial de Santander. Bucaramanga, Colombia. Grupo Variaciones Anatómicas y Biomecánica Tendomuscular. 


\section{MATERIAL Y MÉTODO}

Se disecó el estribo mediante micro-disección de bloques de hueso temporal obtenidos de especímenes cadavéricos adultos frescos de raza mestiza, a quienes se les practicó autopsia en el Instituto de Medicina Legal de Bucaramanga (Colombia) con un abordaje superior a través de la fosa craneal media. El receso epitimpánico fue removido con motor y expuesta la cavidad ótica media en donde se luxó el martillo, el yunque y el estribo en las articulaciones incudo-maleolar, incudo-estapedial y anular. Se limpiaron con agua oxigenada durante 2 horas y registraron. La toma de medidas en las muestras de estribo las realizo un solo operador y de manera directa en cada hueso, con calibrador electrónico (Mitutoyo®) y microscopia de aumen- to de $12 \mathrm{x}$. Las registros fotográficos fueron logrados con video-microscopio de 50x.

Las zonas medidas en el estribo (Tabla I-III) corresponden espacialmente a la longitud y el ancho de la articulación lenticular, de los procesos anterior y posterior individual y conjuntamente (distancia y angulación) y por ultimo la platina.

Se realizaron medidas paramétricas (promedio, rango y desviación estándar) de cada variable y una prueba T pareada en 22 de los especímenes que correspondieron a muestras izquierdas y derechas de un mismo individuo.

Tabla I. Medidas del estribo en 23 muestras (ver Fig. 1).

\begin{tabular}{lcccccccc}
\hline Medida & Largo Platin & $\begin{array}{c}\text { Ancho } \\
\text { Platin-P }\end{array}$ & $\begin{array}{c}\text { Ancho } \\
\text { Platin-M }\end{array}$ & $\begin{array}{c}\text { Ancho } \\
\text { Platin-A }\end{array}$ & Grosor Plat & $\begin{array}{c}\text { Dist } \\
\text { Interpilar Int }\end{array}$ & $\begin{array}{c}\text { Altura Arco } \\
\text { Alt } \\
\text { Artic-Plat }\end{array}$ \\
\hline Promedio & 2,87 & 1,15 & 1,36 & 1,32 & 0,30 & 1,65 & 1,82 & 3,18 \\
DE & 0,09 & 0,14 & 0,09 & 0,18 & 0,05 & 0,17 & 0,19 & 0,15 \\
Rango & $2,66-3,03$ & $0,89-1,40$ & $1,11-1,47$ & $1,01-1,56$ & $0,2-0,41$ & $1,42-1,99$ & $1,51-2,21$ & $2,93-3,46$ \\
\hline
\end{tabular}

Largo Platin: Longitud antero-posterior de la platina del estribo. (1 en Figura 1)

Ancho Platin-P: Ancho (rostro-caudal) de la porción posterior de la platina del estribo. (2 en Figura 1)

Ancho Platin-M: Ancho (rostro-caudal) de la porción media de la platina del estribo. (3 en Figura 1)

Ancho Platin-A: Ancho (rostro-caudal) de la porción anterior de la platina del estribo. (4 en Figura 1)

Grosor Plat: Grosor de la platina del estribo. (5 en Figura 1)

Dist Interpilar Int: Distancia entre parte interna de la apófisis anterior y posterior del estribo. (6 en Figura 1)

Altura Arco: Distancia entre el arco y la platina del estribo. (7 en Figura 1)

Alt Artic-Plat: Distancia entre la articulación lenticular a la superficie de la platina del estribo. (8 en Figura 1)

Tabla II. Medidas del estribo en 23 muestras (ver Figura 2).

\begin{tabular}{lccccccc}
\hline Medida & Artic A-P & Artic R-C & Anc Pilar Ant & Gr Pilar Ant & Anc Pilar Post & Gr Pilar Post & Dist Interpilar Ext \\
\hline Promedio & 1,18 & 0,90 & 0,36 & 0,48 & 0,49 & 0,61 & 2,50 \\
DE & 0,11 & 0,11 & 0,07 & 0,08 & 0.12 & 0.11 & 0.37 \\
Rango & $0,96-1,39$ & $0,63-1,05$ & $0,24-0,5$ & $0,28-0,6$ & $0,31-0,76$ & $0,41-0,75$ & $1,04-1,93$ \\
\hline
\end{tabular}

Artic A-P: Longitud antero-posterior de la articulación lenticular del estribo. (1 en Figura 2)

Artic R-C: Longitud rostro-caudal de la articulación lenticular del estribo. (2 en Figura 2

Anc Pilar Ant: Ancho rostro-caudal del pilar anterior del estribo. (3 en Figura 2)

Gr Pilar Ant: Grosor antero-posterior del pilar anterior del estribo. (4 en Figura 2)

Anc Pilar Post: Ancho rostro-caudal del pilar posterior del estribo. (5 en Figura 2)

Gr Pilar Post: Grosor antero-posterior del pilar posterior del estribo. (6 en Figura 2)

Dist Interpilar Ext: Distancia entre parte externa de la apófisis anterior y posterior del estribo. (7 en Figura 2)

Tabla III. Ang Converg Crural: Ángulo formado entre la apófisis anterior y posterior del estribo.

\begin{tabular}{ll}
\hline Medida & Ang Converg Crural \\
Promedio & 29,01 \\
Desviación Estándar & 2,93 \\
Rango & $25,26-34,88$ \\
\hline
\end{tabular}




\section{RESULTADOS}

En las Tablas I, II y III, se encuentran las medidas pormenorizadas para cada registro de los veintitrés especímenes de estribo estudiadas, con sus promedios y dis- persiones y rangos individuales. Correspondientes con las tablas, las Figuras 1, 2 y 3 muestran los sitios para cada registro.
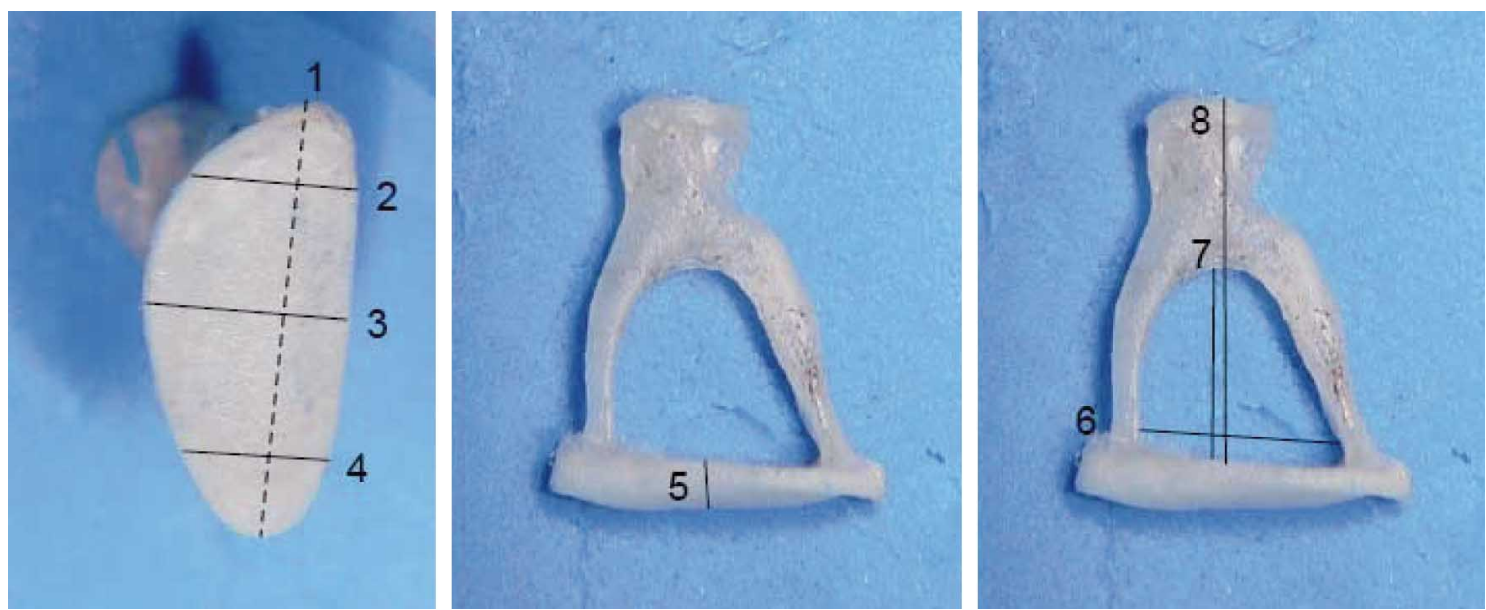

Fig. 1. Medidas del estribo en platina, distancias internas del arco intercrural y longitud total. Nomenclatura en tabla.
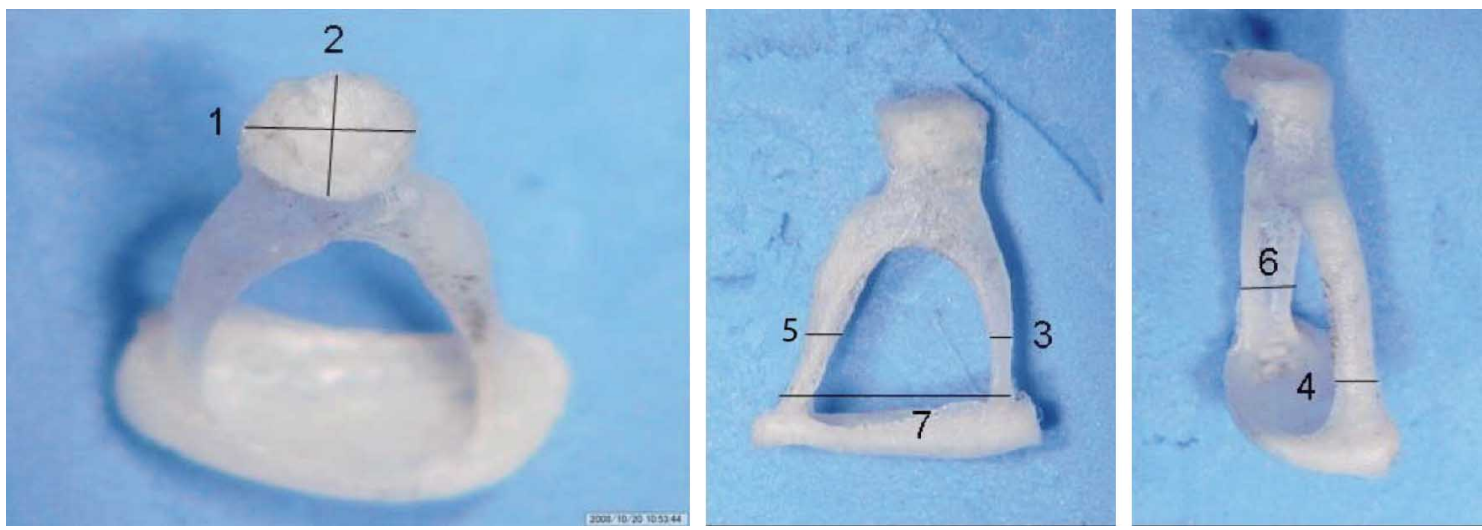

Fig. 2. Medidas del estribo en carilla lenticular, distancia eterna del arco intercrural y procesos anterior y posterior. Nomenclatura en tabla.

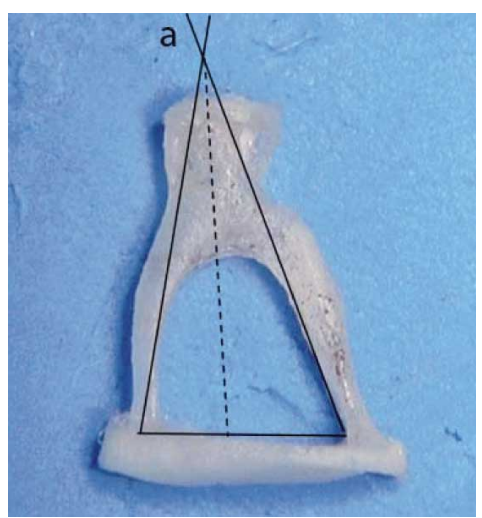

Fig. 3. Angulo formado entre las inclinaciones de las cruras del estribo (a)
La medida angular intercrural (Fig. 3) se encontró a partir de los catetos de un triangulo formado de la longitud total vertical del estribo y de la longitud horizontal intercrural. Se encontró dividiendo el cateto opuesto (longitud vertical del estribo) sobre el cateto adyacente (longitud intercrural). Este ángulo obtenido (de una sola crura) se multiplicó por dos (dos cruras). 


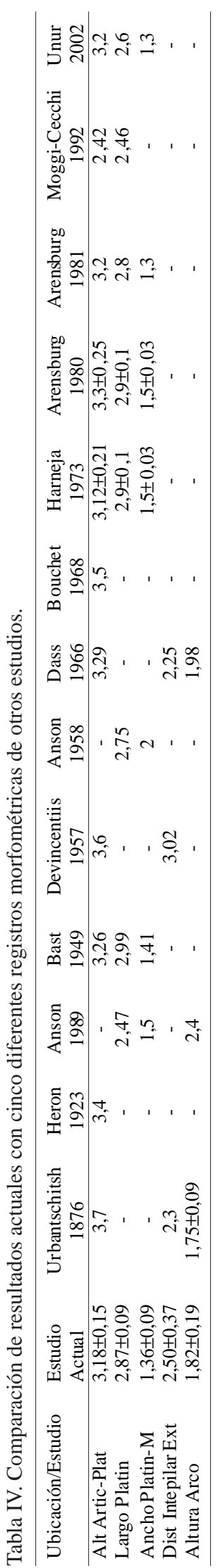

\section{DISCUSIÓN}

La estructura, función, morfometría y peso de los huesecillos del oído medio en fetos, adultos y niños han sido estudiados sin mostrar importantes variaciones (Olsewski, 1989; Bruintje, 1990; Olsewski, 1990; Anson \& Donaldson 1991). Los modelos matemáticos han sido de mucha utilidad en su estudio y en la reproducción de su mecánica (Wassif, 1951; Arensburg \& Nathan, 1971; Tonndorf \& Khanna, 1972; Sarrat et al.; Proop et al., 1984; 1988; Aycan et al., 1990; Mills, 1991; Nishihara \& Goode, 1994; EWengen et al., 1995; Asai et al., 1999; Sun et al., 2002, Whyte et al., 2003; Morris et al., 2004; Murugasu et al., 2005; Farahani \& Nooranipour, 2008).

De las medidas registradas en este estudio, las longitudes comparables con otros anteriores (Tabla 4) muestran similitudes en su gran mayoría con los primeros estudios con algunas diferencias ocasionales que responden probablemente al instrumento de medición usado es su registro.

En relación a variaciones, nuestros resultados coinciden con la percepción general de que el estribo es un hueso muy estable sin presencia de importantes variaciones (n et al., 1992; Unur et al., 1993), incluidos los cambios postnatales (Olsewski, 1989; Olsewski, 1990). Arensburg et al. (1981) encuentran los oscículos con morfometría muy estables en poblaciones históricas y prehistóricas con contrastes étnicos, temporales (miles de años) y geográficos amplios. Coincidente, Crevecoeur (Crevecoeur, 2007) estudia la morfometría y la relación filogenética del homínido hombre de diferentes épocas evolutivas midiendo los oscículos desde antes del Neandertal, pasando por el paleolítico y llegando hasta el humano moderno, concluyendo que estos huesecillos son morfométricamente muy estables tanto en poblaciones prehistóricas como históricas, como aquellas étnica y geográficamente diferentes. Este autor matiza que el tamaño del cráneo y el cuerpo no guardan correlación con el de los oscículos, lo que tiene un valor filogenético sustancial. Esto se debe traducir en muy pocas variaciones anatómicas para estas estructuras óseas. Quam (Quam \& Rak, 2008) no encuentran diferencias morfológicas significativas salvo algunas diferencias morfométricas sutiles entre oscículos de hombre moderno y Neandertal que asocian a la evolución.

En la obtención del ángulo intercrural a partir de la fórmula usada en este estudio, es esencial la simetría de lados al eje mayor del estribo, situación demandante pero realizable debido a que el estribo es el hueso más simétrico de los tres oscículos. Farahani y Col. (Farahani \& Nooranipour, 2008) realizaron la medición de este ángulo con microscopia electrónica en 12 estribos obteniendo un ángulo promedio de 19.5 grados DE 2.3. Revisando la literatura no se encontró más información sobre esta variable que permitiera enriquecer la discusión.

El registro de todas las medidas óseas del estribo buscó corroborar y aportar a lo encontrado en otras investigaciqones. De esta forma se amplía la información desde una perspectiva étnica y geográfica diferente ya que no hay trabajos parecidos en población mestiza Colombiana. Finalmente un análisis pareado de estas medidas encontró que no hubo diferencia de lado en los registros.

\section{CONCLUSIONES}

En términos generales encontramos que la morfología del estribo es homogénea y estable. En las muestras pareadas no encontramos diferencias significativas de lado. 
RAMIREZ, L. M. \& BALLESTEROS, L. E. Human's stape anthropometry: A direct anatomic study. Int. J. Morphol., 30(4):12951300, 2012.

SUMMARY: There is a modest database about morphological data for the stapes in humans. Its preliminary characterization is important when compared with other ancestral times and also in the study of a complex oscicular biomechanics. Paired and structural characteristics of the stapes were registered in 23 bones of Colombian adult mestizo population. Registered measures involved the lenticular incudo-stapedial joint; the anterior and posterior processes of the stapes; and the footplate dimensions. Five of the sixteen records taken in this study could be carefully compared to other results. The five comparable measures were: the total length of the footplate with $2.87 \mathrm{SD} 0.09 \mathrm{~mm}$; the widest footplate zone with $1.36 \mathrm{SD} 0.09 \mathrm{~mm}$; the stape's total length with $0.15 \mathrm{SD} 3.18$ and internal interprocesses length with $1.65 \mathrm{SD} 0.17$; and the arc height with $1,82 \mathrm{SD} 0,19$. Anterior and posterior angulation of the stape processes was 29.01 SD 2.93 degrees. In accordance with the studied population the measures cannot show individually or paired variations in the anatomy of the stapes, making it a uniform and morphometrically stable bone.

KEY WORDS: Oscicles, stapes, footplate, lenticular joint, incudo-stapedial joint, anterior process, posterior process.

\section{REFERENCIAS BIBLIOGRÁFICAS}

Anson, B. J. \& Donaldson, J.A. Surgical anatomy of the temporal bone. $3^{\text {a }}$ ed. Philadelphia WB Saunders, 1991.

Arensburg, B. \& Nathan, H. Observations on a notch in the short (Superior or Posterior) process of the incus. Acta Anat., 78:8490, 1971.

Arensburg, B.; Harell, M. \& Nathan, H. The human middle ear ossicles, morphometry and taxonomic implications. J. Hum. Evol., 10:199-205,1981.

Asai, M.; Heiland, K. E.; Huber, A.M. \& Goode, R.L. Evaluation of cement incus replacement prosthesis in a temporal bone model. Acta Otolaryngol., 119:573-6, 1999.

Aycan, K.; Unur, E. \& Bozkır, M.G. Anatomical study of malleus. J. Health Sciences., 1:152-58, 1990.

Bruintje Tj.D. The auditory ossicles in human skeletal remains from a leper cemetery in Chichester, England. J. Archaeological Science, 17: 627-33, 1990.

Cole., F.G. In History of comparative anatomy. London, Macmillan \& Co. Ltd., 69-120, 1949.

EWengen, D.F., Nishihara, S., Kurokawa, H. \& Goode R.L. Measurements of the stapes superstructure. Ann. Otol. Rhinol. Laryngol., 104:311-6, 1995.

Farahani, R.M. \& Nooranipour, M. Anatomy and anthropometry of human stapes. Am. J. Otolaryngol., 29:42-7, 2008.

Gillespie, P.G. \& Muller U. Mechanotransduction by hair cells: models, molecules, and mechanisms. Cell, 139:33-44, 2009.

Guyton. \& Hall.Tratado de Fisiología Médica $11^{\circ}$ Ed. Elsevier, 2007.
Harneja, N. K., \& Chaturvedi R.P. A study of the human ear ossicles. Indian J. Otolaryng Head \& Neck Surg, 25:154-60, 1973.

Heron, I. C. Measurements and observations upon tile human auditory ossicles. Am. J. Physic. Anthrop., 6:11-26, 1923.

Mills, R. P. Ossicular geometry and the choice of technique for ossiculoplasty. Clin. Otolaryngol. Allied Sci., 16:476-9, 1991.

Moggi-Cecchi J, Collard M. A fossil stapes from Sterkfontein, South Africa, and the hearing capabilities of early hominids. J. Hum. Evol., 42:259-65, 2002.

Morris, D. P.; Bance, M.;van Wijhe, R.G.; Kiefte, M. \&Smith, R. Optimum tension for partial ossicular replacement prosthesis reconstruction in the human middle ear. Laryngoscope, 114:305-8, 2004.

Murugasu, E.; Puria, S. \& Roberson, J.B. Malleus-to-footplate versus malleus-to-stapes-head ossicular reconstruction prostheses: temporal bone pressure gain measurements and clinical audiological data. Otol. Neurotol., 26:572-82, 2005.

Nishizaki, K. \& Anniko, M. Developmental morphology of the middle ear. Auris Nasus Larynx., 24:31-8, 1997.

Nishihara, S. \& Goode, R.L. Experimental study of the acoustic properties of incus replacement prostheses in a human temporal bone model. Am. J. Otol., 15:485-94, 1994.

Olsewski, J. Structure of the middle ear in infants. Otolaryngol. Pol., 43:278-83, 1989.

Olsewski, J. The morphometry of the ear ossicles in humans during development. Anat Anz., 171:187-91, 1990.

Proop, D.; Hawke, M. \& Berger, G. The anterior process of the malleus. J. Otology, 6:257-62, 1984. 
Ramirez, L. M. \& Ballesteros, L. E. Human oscicular chain articulations: Asymmetric Sound Transmission. Int. J. Morphol., 28:1059-68, 2010.

Rodriguez Vazquez, J.F., Merida, V., Jr. \& Jimenez, C.J. A study of the os goniale in man. Acta Anat (Basel)., 142:188-92, 1991.

Rodriguez Vazquez, J. F., Merida, V. Jr. \& Jimenez, C. J. Relationships between the temporomandibular joint and the middle ear in human fetuses. J. Dent Res., 72:62-6, 1993.

Sarrat, R.; Guzman, G. \& Tores, A. Morphological variations of Human ossicular tympani. Acta Anat., 131:146-9, 1988.

Sarrat, R.; Torres, A.; Guzman, A.G.; Lostalé, F. \& Whyte, J. Functional structure of human auditory ossicles. Acta Anat. (Basel)., 144:189-95,1992.

Sun, Q.; Gan, R.Z.; Chang, K.H. \& Dormer, K.J. Computerintegrated finite element modeling of human middle ear. Biomech. Model. Mechanobiol., 1:109-22, 2002.

Tonndorf, J.\& Khanna,S.M. Tympanic-membrane vibrations in human cadaver ears studied by time-averaged holography. $J$. Acoust. Soc. Am., 52:1221-33, 1972.

Unur, E.; Aycan, K. \& Ekinci, N. The study of incus from morphometric view. Erciyes Medical J., 24:57-63, 2002.

Wassif, K. The anterior process and the ossification of the malleus in mammals. Science., 27:113:486-7, 1951.

Whyte, J.; Cisneros, A.I.; Urieta, J.J.; Yus, C.; Gañet, J.; Torres, A. \& Sarrat, R. Ontogenic peculiarities of the human tympanic ossicular chain. Acta Otorrinolaringol Esp., 54:1-10, 2003.

33. Whyte, J.R., González, L., Cisneros, A.I., Yus, C., Torres, A., Sarrat, R. Fetal development of the human tympanic ossicular chain articulations. Cells Tissues Organs, 171:241-9, 2002.

Willi, U.B., Ferrazzini, M.A., Huber, A.M. The incudo-malleolar joint and sound transmission losses. Hear Res., 174:32-44, 2002.
Dirección para correspondencia:

Luis Miguel Ramirez Aristeguieta, DDS, MS, MSc

Profesor Asociado Facultad de Odontologia

Universidad de Antioquia

COLOMBIA

Email: Imra3@yahoo.com

Recibido : 17-04-2012

Aceptado: 07-08-2012 